\title{
On the Discrepancy of Quasi-progressions
}

\author{
Sujith Vijay \\ Department of Mathematics \\ Rutgers, The State University of New Jersey, USA* \\ sujith@math.rutgers.edu
}

Submitted: Apr 30, 2006; Accepted: Aug 1, 2008; Published: Aug 11, 2008

Mathematics Subject Classification: 05C65, 11B25

\begin{abstract}
A quasi-progression, also known as a Beatty sequence, consists of successive multiples of a real number, with each multiple rounded down to the largest integer not exceeding it. In 1986, Beck showed that given any 2-colouring, the hypergraph of quasi-progressions contained in $\{0,1, \ldots, n\}$ corresponding to almost all real numbers in $(1, \infty)$ have discrepancy at least $\log ^{*} n$, the inverse of the tower function. We improve the lower bound to $(\log n)^{1 / 4-o(1)}$, and also show that there is some quasi-progression with discrepancy at least $(1 / 50) n^{1 / 6}$. The results remain valid even if the 2-colouring is replaced by a partial colouring of positive density.
\end{abstract}

\section{Introduction}

A classic result in discrepancy theory is the theorem of Roth [2] stating that if the elements of $\{0,1,2, \ldots, n\}$ are 2-coloured (e.g: red/blue), there exists an arithmetic progression $\{a, a+d, \ldots, a+(k-1) d\}$ of discrepancy (i.e., difference in the number of reds and blues) at least $(1 / 20) n^{1 / 4}$, with $0 \leq a<d \leq \sqrt{6 n}$. In 1996, Matoušek and Spencer [10], building upon results by Sárközy (see [3]) and Beck [5], showed that apart from constants, this result is the best possible.

The situation is quite different, however, for homogeneous arithmetic progressions (HAPs), the subfamily of arithmetic progressions containing 0 . It turns out that there are extremely balanced colourings for such arithmetic progressions. Consider the following examples.

$$
\begin{aligned}
& \chi_{3}(3 k+1)=1 ; \chi_{3}(3 k+2)=-1 ; \chi_{3}(3 k)=\chi_{3}(k) \\
& \chi_{3}^{*}(3 k+1)=1 ; \chi_{3}^{*}(3 k+2)=-1 ; \chi_{3}^{*}(3 k)=0
\end{aligned}
$$

${ }^{*}$ Current Address: Department of Mathematics, University of Illinois at Urbana-Champaign, Urbana, IL 61801. E-mail: sujith@math.uiuc.edu 
It is easy to show that all HAPs contained in $\{0,1, \ldots, n\}$ have discrepancy $O(\log n)$ under the colouring $\chi_{3}$ and discrepancy at most 1 under the partial colouring $\chi_{3}^{*}$. In general, for any prime $p$, we can define a partial colouring $\chi_{p}^{*}$ via the non-trivial real character modulo $p$ (the Legendre symbol), and the resulting discrepancy of HAPs would be bounded by $(p-1) / 2$. Also note that $\chi_{p}^{*}$ is "almost admissible" for large $p$, since only a small fraction of numbers is coloured 0. Whether there is a "completely admissible" colouring of bounded discrepancy for HAPs is a question raised by Erdös in the 1930s, and one that remains unsolved to this day. It is indeed a mishap that this innocent-looking question should turn out to be so difficult. We study a variant of this problem.

A quasi-progression $Q(\alpha ; s, t)$ is the sequence of integers

$$
\lfloor s \alpha\rfloor,\lfloor(s+1) \alpha\rfloor, \ldots,\lfloor t \alpha\rfloor
$$

In other words, a quasi-progression is a sequence of successive multiples of a real number, with each multiple rounded down to the largest integer below it. Note that for integer values of $\alpha$, quasi-progressions reduce to HAPs, or the set-difference of two HAPs. Thus the problem raised by Erdős concerns a subfamily of quasi-progressions, corresponding to integer values of $\alpha$.

\section{Lower Bounds}

Our first theorem gives a lower bound on the discrepancy of the family of all quasiprogressions contained in $\{0,1, \ldots, n\}$.

Theorem 1 If the integers from 0 to $n$ are 2-coloured, there exists $\alpha>1$ and integers $s$ and $t$ such that the quasi-progression $Q(\alpha ; s, t)$ has discrepancy at least $(1 / 50) n^{1 / 6}$.

Proof Let $m<n$. The value of $m$ will be specified at the end of the proof. By Roth's theorem, there exists an arithmetic progression $\mathcal{P}=\{a, a+d, a+2 d, \ldots\}$ contained in $\{n-m, n-m+1, \ldots, n\}, 2 \leq d<\sqrt{6 m}, 0 \leq a<d$, with discrepancy at least $(1 / 40) m^{1 / 4}$. We will show that for suitably chosen $m, \mathcal{P}$ can be realised as a quasi-progression corresponding to a real number $\alpha>1$.

Observe that if $\alpha=d-\epsilon$, the first $\lfloor 1 / \epsilon\rfloor$ elements in the sequence $\lfloor\alpha\rfloor,\lfloor 2 \alpha\rfloor,\lfloor 3 \alpha\rfloor \ldots$ are congruent to $-1(\bmod d)$, the next $(\lfloor 2 / \epsilon\rfloor-\lfloor 1 / \epsilon\rfloor)$ elements are congruent to $-2(\bmod d)$, and so on. In particular, the arithmetic progression $\mathcal{P} \equiv-(d-a)(\bmod d)$ can be realised as a quasi-progression by choosing $\epsilon$ such that $\mathcal{P}$ is completely contained in the $(d-a)^{t h}$ block of length $\lfloor 1 / \epsilon\rfloor$ or $\lceil 1 / \epsilon\rfloor$, as the case may be.

Since $\mathcal{P} \subseteq\{n-m, n-m+1, \ldots, n\}$, it suffices to choose $\epsilon$ such that $\lceil x \epsilon\rceil=d-a$ for 
$n-m+1 \leq x \leq n$. Such an $\epsilon$ exists if

$$
\frac{n-m+1}{d-a-1}>\frac{n}{d-a}
$$

Note that $d-a \leq d \leq \sqrt{6 m}$. Therefore, we can choose $m=\left\lfloor 6^{-1 / 3} n^{2 / 3}\right\rfloor$. This yields a quasi-progression of discrepancy at least $(1 / 50) n^{1 / 6}$.

While it is not known whether the set of homogeneous arithmetic progressions have bounded discrepancy, there exist colourings (see [11]) for which the arithmetic progression $\{0, d, 2 d, \ldots$,$\} has discrepancy at most d^{4+o(1)}$ for all $d$. It turns out, however, that upper bounds independent of $n$ do not exist for most quasi-progressions.

Let $\alpha>1$ be given, together with a 2 -colouring of $\{0,1, \ldots, n\}$. Let $D_{\alpha}(n)$ denote the maximum discrepancy of $Q(\alpha ; s, t)$ over all admissible $s$ and $t$. In 1986, Beck [6] showed that given any 2-colouring of the non-negative integers, for almost every $\alpha \in[1, \infty)$, there are infinitely many $n$ such that $D_{\alpha}(n) \geq \log ^{*} n$. Recall that $\log ^{*} x$ denotes the inverse of the tower function: $\log ^{*} x=\ln x$ for $1<x<e$ and $\log ^{*}\left(e^{x}\right)=1+\log ^{*} x$.

We improve on this result, and prove the following theorem.

Theorem 2 Let $\chi$ be a partial colouring of the non-negative integers with density $\rho>0$, and let $\chi_{n}$ denote the restriction of $\chi$ to $\{0,1, \ldots, n\}$. Then for almost every $\alpha \in[1, \infty)$, there are infinitely many $n$ such that $D_{\alpha}(n) \geq(\log n)^{1 / 4-o(1)}$.

Proof We begin with a definition.

Definition: We say that the real number $\alpha$ is $M$-balanced if $D_{\alpha}(n) \leq M$. For brevity, we shall hereafter refer to $\left(c_{0}(\log n)^{1 / 4}\right)$-balanced $\alpha$ simply as "balanced".

Let $E$ denote the set of $\alpha$ such that there are only finitely many $n$ with $D_{n}(\alpha) \leq$ $(\log n)^{1 / 4-o(1)}$ under the colouring $\chi$. If $E$ has positive measure, there exists a positive integer $t$ for which the set of balanced $\alpha$ in $[t, t+1)$ has measure $\delta>0$. But it follows from the Main Lemma (see below) that there exists $c_{0}=c_{0}(\rho, t, \delta)$ such that the set of $\alpha$ with $D_{n}(\alpha) \leq c_{0}(\log n)^{1 / 4}$ has measure less than $\delta$. For all other $\alpha$ in $[t, t+1)$, we have $D_{n}(\alpha)>(\log n)^{1 / 4-o(1)}$ for sufficiently large $n$, yielding a contradiction.

It remains to state and prove the Main Lemma.

Main Lemma Let $\chi$ and $\chi_{n}$ be as in the statement of Theorem 2. Given $t \in[1, \infty)$ and $\delta>0$, there exists $c_{0}=c_{0}(\rho, t, \delta)$ such that the set of $\alpha$ in $[t, t+1)$ with the property that $D_{\alpha}(n) \leq c_{0}(\log n)^{1 / 4}$ under $\chi_{n}$ for all $n$, has Lebesgue measure less than $\delta$.

Proof We will transform the problem into a geometric setting, with a view to using orthogonal functions, as was done by Roth [1] in his classic paper on the measure-theoretic 
discrepancy of axis-parallel rectangles. A similar construction was used by Hochberg (see [8] and [9]) to show the existence of a quasi-progression of discrepancy $c_{0}^{\prime}(\log n)^{1 / 4}$. As we saw in Theorem 1, quasi-progressions with much larger discrepancy do occur.

We shall assume, for the sake of convenience, that $n=(t+1) m$ where $m=2^{u}$ for some positive integer $u$. We join each lattice point $(a, b)$ with the one vertically above it, and give the resulting unit segment the colour $\chi(b)$. For each point $(x, y)$ in the plane, the discrepancy function $D(x, y)$ is defined to be the sum of the $\chi$-values of the unit segments crossed by the line joining $(0,0)$ and $(x, y)$. Note that $|D(x, y)| \leq M$ if and only if $y / x$ is $M$-balanced.

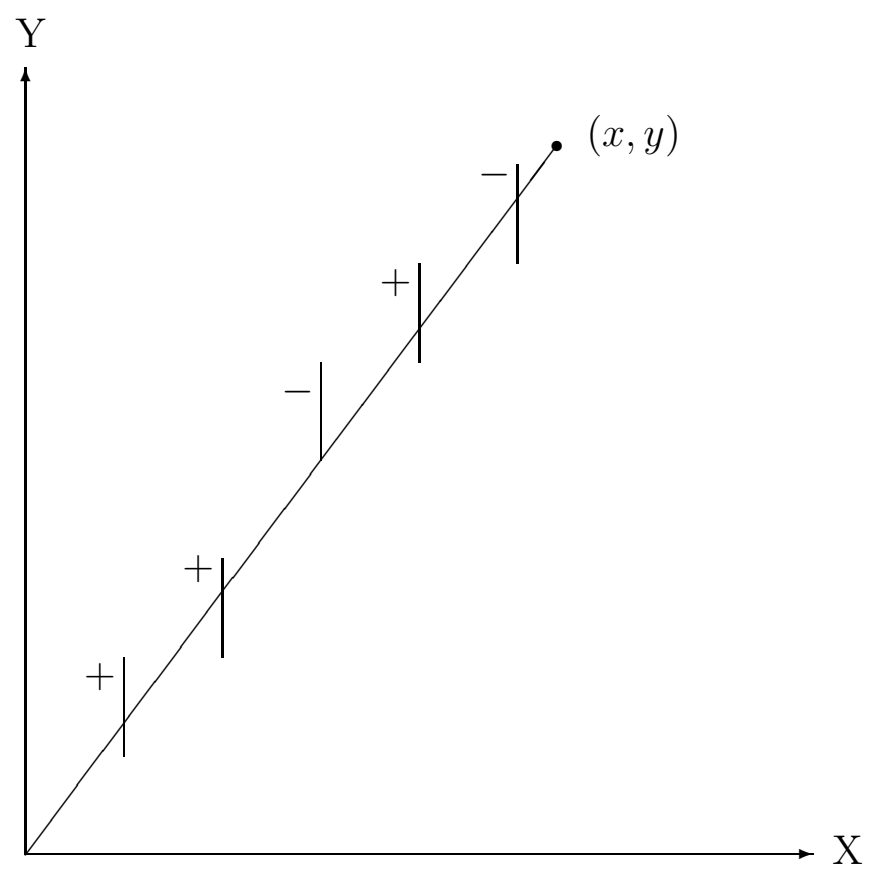

Let $H(x, y)=D(x, y)$ if $y / x$ is balanced, and 0 otherwise. Suppose that the measure of the set of balanced $\alpha$ in $[t, t+1)$ is at least $\delta$. We will deduce a contradiction for a suitably chosen $c_{0}$ by producing a point $\left(x_{0}, y_{0}\right)$ with $H\left(x_{0}, y_{0}\right)>c_{0}(\log n)^{1 / 4}$.

Let $R$ denote the region bounded by the lines $x=m / 2, x=m, y=t x, y=(t+1) x$. We will construct orthonormal functions $g_{1}, g_{2}, \ldots, g_{r}$ on $R$ where $r=(\log n) / 8$ and

$$
\sum_{i=1}^{r}\left(\left\langle H, g_{i}\right\rangle\right)^{2} \geq \frac{\rho^{2} \delta^{13} m^{2}(\log n)^{1 / 2}}{2^{29} c_{0}^{2} t^{3}}
$$

Since $R$ has area $3 m^{2} / 8$, it follows from Bessel's inequality that there exists $\left(x_{0}, y_{0}\right)$ 
with $H\left(x_{0}, y_{0}\right)>c_{0}(\log n)^{1 / 4}$ for

$$
c_{0}(\rho, t, \delta)=\frac{\rho^{1 / 2} \delta^{13 / 4}}{120 t^{3 / 4}}
$$

yielding the desired contradiction.

The functions $g_{1}, g_{2}, \ldots, g_{r}$ will be normalised versions of mutually orthogonal functions $G_{1}, G_{2}, \ldots, G_{r}$. Following Hochberg, we will construct $G_{i}$ by dividing $R$ into a grid of trapezoids, called the $i^{\text {th }}$ trapezoidal grid. We use vertical lines spaced $\ell=2^{i}$ apart and slanting lines with slopes equally spaced between $t$ and $t+1$. The slopes of consecutive slanting lines differ by $\tau \doteq 1 /(\ell \beta m)$ where $\beta=c_{2}(\log n)^{1 / 4}$. The value of $c_{2}$ will be specified later. It is easy to see that the individual grid trapezoids have area at most $1 / \beta$ and at least $1 /(2 \beta)$.

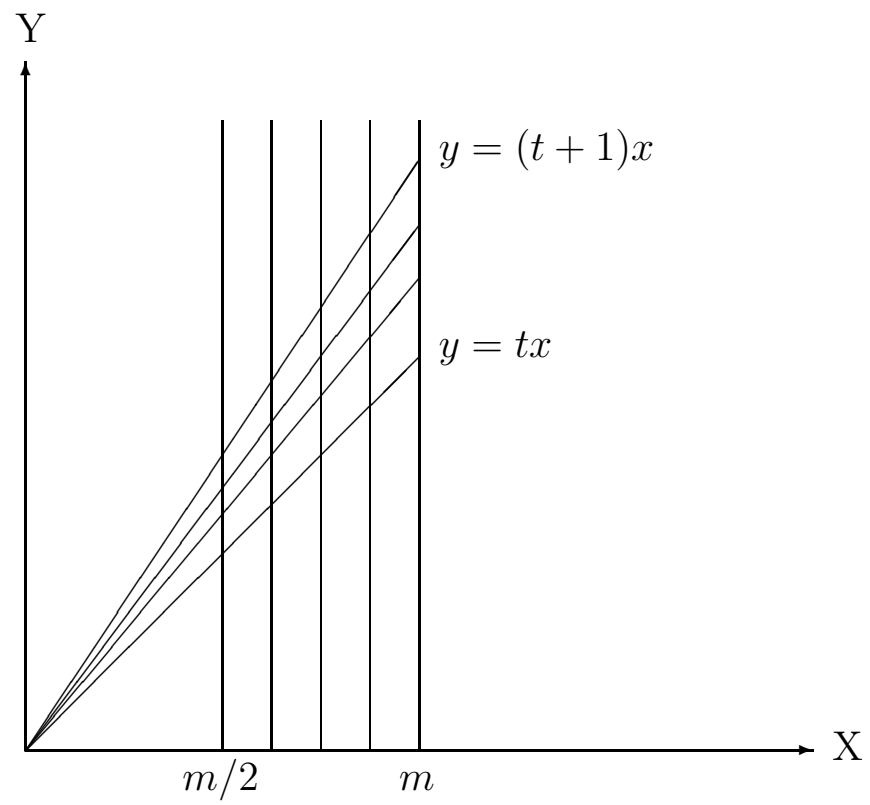

Note that we have specified only the spacing between the grid lines and not their actual position. We choose the position of the rightmost vertical line randomly and uniformly in the interval $[n-\ell, n)$, and the slope of the lowermost line randomly and uniformly in the interval $[t, t+\tau)$. The region between two consecutive sloping lines will be called a sector, and sectors will be identified with subintervals of $[t, t+1)$ in the natural fashion. We will denote the measure of balanced $\alpha$ in the $j^{\text {th }}$ sector of the $i^{\text {th }}$ grid by $\mu_{i j}$. For convenience, we define $\mu_{i j}^{*}=\mu_{i j} / \tau$.

If $\chi(b) \neq \chi(b-1)$, we refer to $b$ as a switch value. Furthermore, a lattice point $(a, b)$ will be called a switch point if $b$ is a switch value. A switch point is said to be good if it finds itself alone in a trapezoid no matter how the grid is positioned; bad otherwise. We shall denote the number of good switch points in the $j^{\text {th }}$ sector of the $i^{\text {th }}$ grid by $s_{i j}^{*}$. 
We define $G_{i}$ as follows: On a trapezoid containing exactly one switch point, $G_{i}$ is defined in a checkerboard fashion. On all other trapezoids, $G_{i}$ is defined to be identically zero.

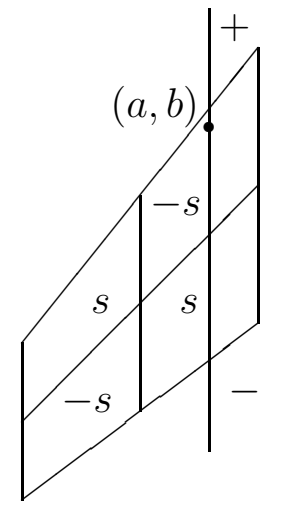

The vertical dividing line passes through the centre of the trapezoid. The position of the slanting dividing line is chosen such that the measure of balanced $\alpha$ above the line and inside the sector equals the measure of balanced $\alpha$ below the line and inside the sector. The value of $s$ will vary from trapezoid to trapezoid, but will always equal +1 or -1 . Since the vertical dividing lines are nested dyadically (note that the vertical spacing is $\ell=2^{i}$, it is clear that $\left\{G_{i}\right\}$ form an orthogonal family.

We now derive a lower bound on the inner product $\left\langle H, G_{i}\right\rangle$. The position of the slanting dividing line has been chosen with a view to extending Hochberg's argument for the $\mu_{i j}^{*}=1$ case to the more general problem at hand.

Lemma $1 E\left(\left\langle H, G_{i}\right\rangle\right) \geq\left(\sum_{j}\left(\mu_{i j}^{*}\right)^{2} s_{i j}^{*}\right) /(64 \beta)$

Proof Consider the contribution of a unit vertical segment $\ell_{a, b}$ joining $(a, b)$ and $(a, b+1)$ to the discrepancy function $H(x, y)$. Let

$$
B_{a, b}=\left\{(x, y): x \geq a, \frac{b}{a} \leq \frac{y}{x}<\frac{b+1}{a}\right\}
$$

denote the set of points behind the line $\ell_{a, b}$. 


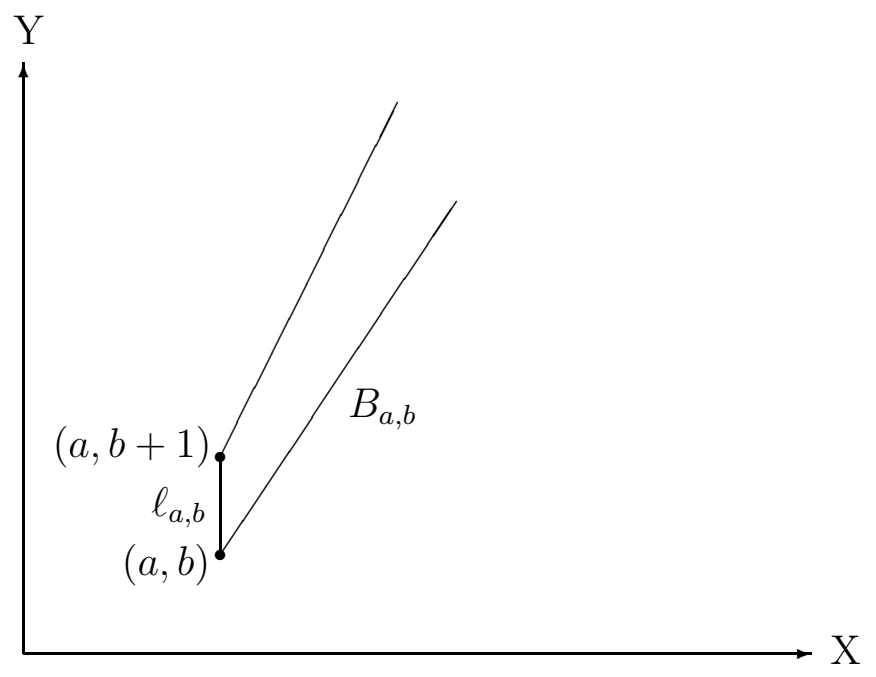

Now define

$$
H_{a, b}(x, y)= \begin{cases}\chi(b) & \text { if }(x, y) \in B_{a, b} \text { and } y / x \text { is balanced } \\ 0 & \text { otherwise }\end{cases}
$$

Clearly,

$$
H(x, y)=\sum_{a=0}^{\infty} \sum_{b=0}^{\infty} H_{a, b}(x, y)
$$

Furthermore, only finitely many terms in this sum are non-zero, for any fixed $(x, y)$. Consider a good switch point $(a, b)$ lying inside a trapezoid $T$, located in the $j^{\text {th }}$ sector of the $i^{\text {th }}$ grid.

We claim that if neither $(a, b)$ nor $(a, b+1)$ lie inside $T$, then

$$
\iint_{T} G_{i}(x, y) H_{a, b}(x, y) d x d y=0
$$

If $T$ lies entirely outside or entirely inside $B_{a, b}$, it is clear that the integral is zero. If exactly one of the bounding lines of $B_{a, b}$ intersects $T$, the geometric symmetry with respect to the vertical dividing line or the measure-theoretic symmetry with respect to the sloping dividing line, as the case may be, ensures that there is perfect cancellation. Thus the integral vanishes in this case as well. 

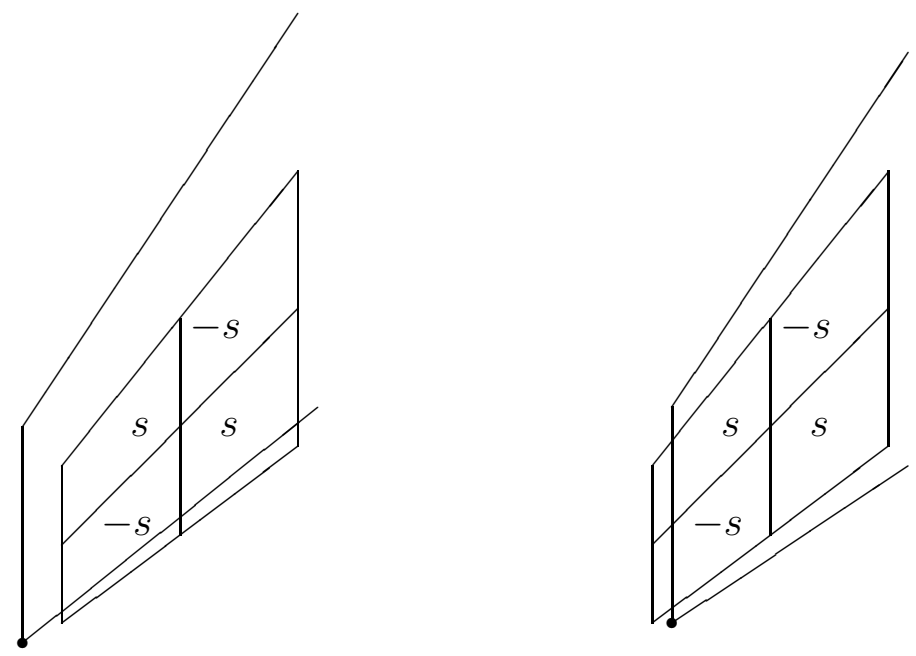

Therefore, we need consider only the terms $H_{a, b}(x, y)$ and $H_{a, b-1}(x, y)$, where $(a, b) \in$ $T$. If $(a, b)$ is not a switch point, we have,

$$
\iint_{T} G_{i}(x, y)\left(H_{a, b}(x, y)+H_{a, b-1}(x, y)\right) d x d y=0
$$

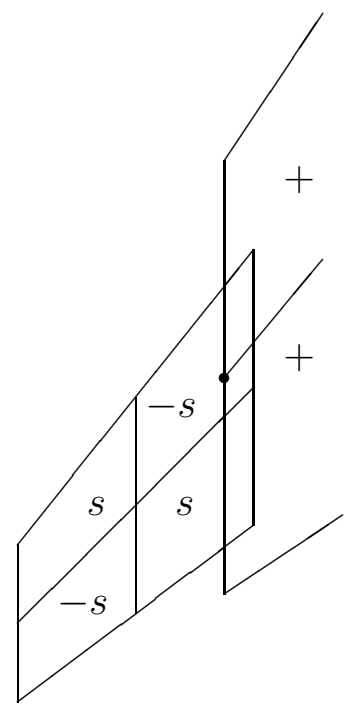

Now suppose that $(a, b)$ is a switch point. If $(a, b)$ lies on the intersection of the two dividing lines, we have

$$
\iint_{T} G_{i}(x, y)\left(H_{a, b}(x, y)+H_{a, b-1}(x, y)\right) d x d y=\frac{s}{4}(\chi(b)-\chi(b-1)) \mu_{i j}^{*} \operatorname{area}(T)
$$




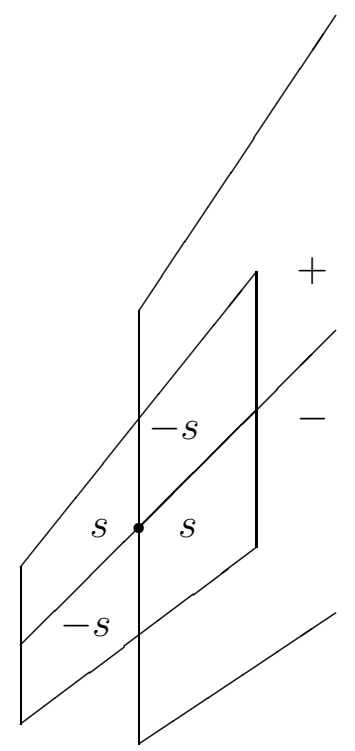

We choose $s$ so that the integral is positive. Since the switch point $p$ is good, there are no other lattice points in $T$, and the value of $s$ can now be safely assumed fixed. Thus we get

$$
\left.\iint_{T} G_{i}(x, y) H(x, y)\right) d x d y \geq \frac{\mu_{i j}^{*}}{8 \beta}
$$

provided $(a, b)$ lies on the intersection of the two dividing lines. The location of $(a, b)$ inside the trapezoid is a uniformly distributed random variable. For the purposes of computing the expectation, we can assume that $T$ is a parallelogram, at the expense of a multiplicative constant. Thus we have

$$
E\left(\iint_{T} G_{i}(x, y) H(x, y) d x d y\right) \geq \frac{\left(\mu_{i j}^{*}\right)^{2}}{64 \beta}
$$

Adding over all switch points and using the linearity of expectation, we get

$$
E\left(\left\langle H, G_{i}\right\rangle\right) \geq \frac{1}{64 \beta} \sum_{j}\left(\mu_{i j}^{*}\right)^{2} s_{i j}^{*}
$$

as claimed.

We now prove a slightly stronger version of a lemma due to Beck [6].

Lemma 2 Let $J \subseteq[0,1]$ be an arbitrary interval of length $\lambda$ and let $1 \leq b_{1}<b_{2}<\ldots b_{q}$ be integers. Let $N(\alpha, J)=\left|\left\{j:\left\{b_{j} \alpha\right\} \in J, 1 \leq j \leq q\right\}\right|$. If $q \geq \lambda^{-6}$, then $\mu(\{\alpha \in[0,1]$ : $N(\alpha, J) \geq(q \lambda / 2)\}) \geq 1-(8 / \sqrt{q})$.

Proof The proof uses LeVeque's inequality from the theory of uniform distributions, and is almost identical to the proof of Beck's original lemma (see [6]). 
Let $x_{j}=\left\{b_{j} \alpha\right\}, 1 \leq j \leq q$. Define $\Delta(\alpha)$ and $S_{n}(\alpha)$ as follows:

$$
\begin{gathered}
\Delta(\alpha)=\sup _{0 \leq a<b \leq 1}\left|\left(\sum_{j: x_{j} \in[a, b)} \frac{1}{q}\right)-(b-a)\right| \\
S_{n}(\alpha)=\frac{1}{q} \sum_{j=1}^{q} e^{2 \pi i n x_{j}}
\end{gathered}
$$

Note that

$$
\int_{0}^{1}\left|S_{n}(\alpha)\right|^{2} d \alpha=\frac{1}{q^{2}} \int_{0}^{1} \sum_{j=1}^{q} \sum_{k=1}^{q} e^{2 \pi i n\left(b_{j}-b_{k}\right) \alpha} d \alpha=\frac{1}{q}
$$

By LeVeque's inequality,

$$
\Delta^{3}(\alpha) \leq \frac{6}{\pi^{2}} \sum_{n \in N} \frac{\left|S_{n}(\alpha)\right|^{2}}{n^{2}}
$$

Therefore,

$$
\int_{0}^{1} \Delta^{3}(\alpha) d \alpha \leq \frac{6}{\pi^{2}} \int_{0}^{1}\left(\sum_{n \in \mathbb{N}} \frac{1}{n^{2}}\left|S_{n}(\alpha)\right|^{2}\right) d \alpha=\frac{1}{q}
$$

Let $E=\{\alpha \in[0,1): N(\alpha, J) \geq q \lambda / 2\}$ and $F=[0,1) \backslash E$. Clearly,

$$
\frac{\lambda^{3} \mu(F)}{8} \leq \int_{0}^{1}\left|\left(\sum_{j: x_{j} \in J} \frac{1}{q}\right)-\lambda\right| d \alpha \leq \int_{0}^{1} \Delta^{3}(\alpha) d \alpha
$$

Therefore, $\lambda^{3} \mu(F) / 8 \leq 1 / q$. Since $q \geq \lambda^{-6}$, we have

$$
\mu(E)=1-\mu(F) \geq 1-\frac{8}{\sqrt{q}}
$$

proving the lemma.

Let $b_{1}, b_{2}, \ldots, b_{q}$ be the switch values of the colouring $\chi$ in $[N / 2, N]$. Note that $q \geq(N \rho) /\left(4 c_{0}(t+1)(\log n)^{1 / 4}\right)=(m \rho) /\left(4 c_{0}(\log n)^{1 / 4}\right)$. Since switch points come in rows, it is clear that $\chi_{n}$ gives rise to $m q$ switch points.

Lemma $3 \sum_{j}\left(\mu_{i j}^{*}\right)^{2} s_{i j}^{*} \geq \delta^{5} m q /(4096 t)$, for $1 \leq i \leq r$

Proof We say that a sector is rich if $\mu_{i j}^{*}>\delta / 2$. Let $\left\{I_{k}\right\}_{k=1}^{L}$ be an enumeration of the rich sectors. Since $(\delta / 2)(n \ell \beta-L)+L \tau \geq \delta$, we have $L>(\delta / 2) m \ell \beta$.

We use Lemma 2 with $J=[0, \delta /(4 \ell \beta(t+1)]$, so that $\lambda=\delta /(4 \ell \beta(t+1))$. Since $r=\log m / 8$ and $i \leq r$, we have $q \geq \lambda^{-6}$. For an arbitrary interval $I=\left[a_{k}, b_{k}\right)$, let $I^{\prime}$ and 
$I^{\prime \prime}$ denote $\left[a_{k}, c_{k}\right)$ and $\left[c_{k}, b_{k}\right)$ respectively, where the measure of balanced $\alpha$ in $I^{\prime}$ and $I^{\prime \prime}$ are equal. Let $A=\cup_{k=1}^{L} I_{k}^{\prime}$. Note that $A$ has measure at least $\delta^{2} / 8$. Let $B=\{\theta: 1 / \theta \in A\}$. Since $A \subseteq[t, t+1) \subseteq[t, 2 t)$, the measure of $B$ is at least $\delta^{2} /\left(32 t^{2}\right)$.

Let $B^{*}=\{\theta \in B: N(\theta, J) \geq(q \lambda) / 2\}$. For sufficiently large $n, B^{*}$ has measure at least $\delta^{2} /\left(64 t^{2}\right)$. Note that $\theta \in B^{*} \Rightarrow 1 / \theta \in I_{k}^{\prime}$ for some $k$. Suppose $\left\{b_{v} \theta\right\} \in J$ for such a $\theta$. Let $a_{v}=\left\lfloor b_{v} \theta\right\rfloor$. Then we have,

$$
0<\frac{b_{v}}{a_{v}}-\frac{1}{\theta}<\frac{2 \lambda(t+1)}{m}<\frac{\delta \tau}{4}
$$

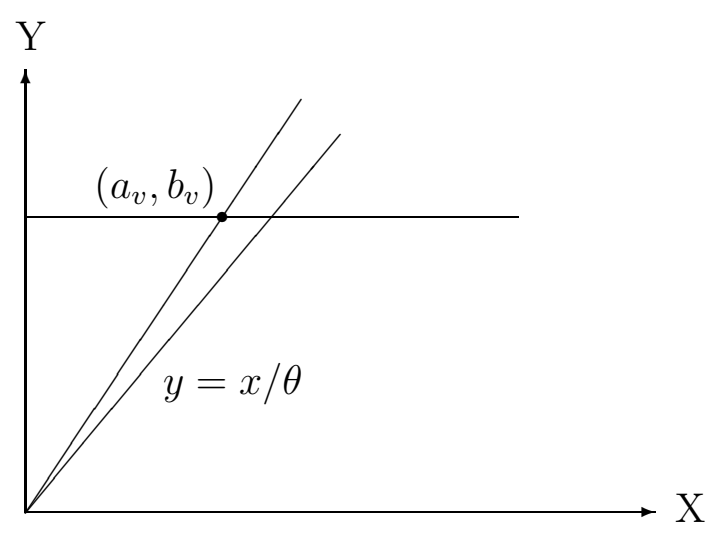

It follows that $\left(b_{v} / a_{v}\right) \in I_{k}$. Thus the $k^{\text {th }}$ sector contains a switch point of the form $\left(a_{v}, b_{v}\right)$.

Since the contribution of a single $I_{k}^{\prime}$ towards the measure of $B^{*}$ is at most $\tau / t^{2}$, there must be at least $\delta^{2} m \ell \beta /\left(32 t^{2}\right)$ rich sectors contributing at least $\delta^{3} m q /(1024 t)$ switch points between them.

We now derive an upper bound on the total number of bad switch points.

Given a bad switch point $(a, b)$, there exists $a^{\prime}$ such that

$$
\left|a^{\prime}-a\right| \leq \ell \text { and }\left\|\frac{b a^{\prime}}{a}\right\|<\frac{1}{\ell \beta}
$$




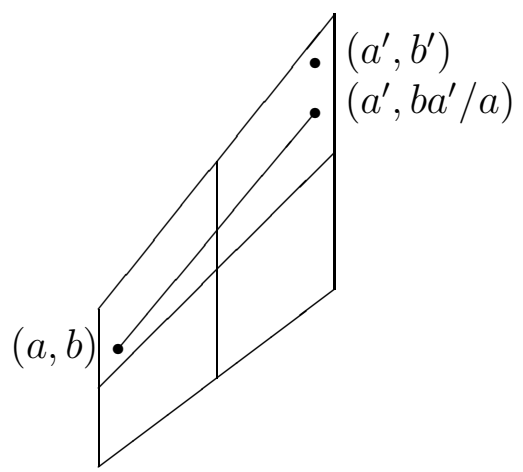

Let $d=\left|a^{\prime}-a\right|$. Note that there are $m \ell / 2$ pairs $(a, d)$ with $1 \leq d \leq \ell, m / 2 \leq a \leq m$. For each such pair, there are $a /(\ell \beta)+O(\ell)$ values of $b$ that satisfy $\|b d / a\|<1 /(\ell \beta)$. It follows that there are at most $m^{2} / \beta$ lattice points which do not find themselves alone in a trapezoid for some placement of the grid.

Since the number of bad switch points is at most

$$
\frac{m^{2}}{\beta}=\frac{m^{2}}{c_{2}(\log n)^{1 / 4}}<\frac{\delta^{3} m q}{1024 t}
$$

for $c_{2} \geq 4096 c_{0} t /\left(\delta^{3} \rho\right)$, we have

as required.

$$
\sum\left(\mu_{i j}^{*}\right)^{2} s_{i j}^{*}>\frac{\delta^{5} m q}{4096 t}
$$

Note that

$$
E\left(\left\langle H, G_{i}\right\rangle^{2}\right) \geq\left[E\left(\left\langle H, G_{i}\right\rangle\right)\right]^{2} \geq\left(\sum_{j} \frac{\left(\mu_{i j}^{*}\right)^{2} s_{i j}^{*}}{64 \beta}\right)^{2}
$$

Furthermore, the combined area of all the grid trapezoids containing exactly one switch point is at most $m q / \beta$. Therefore,

$$
E\left(\left\langle H, g_{i}\right\rangle^{2}\right) \geq \frac{\left(\sum_{j}\left(\mu_{i j}^{*}\right)^{2} s_{i j}^{*}\right)^{2}}{4096 m q \beta} \geq \frac{\rho^{2} \delta^{13} m^{2}}{2^{26} c_{0}^{2} t^{3}(\log n)^{1 / 2}}
$$

By the linearity of expectation,

$$
E\left(\sum_{i=1}^{r}\left\langle H, g_{i}\right\rangle^{2}\right) \geq \frac{\rho^{2} \delta^{13} m^{2}(\log n)^{1 / 2}}{2^{29} c_{0}^{2} t^{3}}
$$

Thus, for some placement of the grids, the resulting $g_{i}$ satisfy

$$
\sum_{i=1}^{r}\left(\left\langle H, g_{i}\right\rangle\right)^{2} \geq \frac{\rho^{2} \delta^{13} m^{2}(\log n)^{1 / 2}}{2^{29} c_{0}^{2} t^{3}}
$$

yielding the statement of the main lemma. 


\section{Upper Bounds}

We derive an upper bound of $O\left(N^{1 / 3+\varepsilon}\right)$ on the discrepancy of quasi-progressions contained in $\{0,1,2, \ldots, N\}$. We begin with an easier estimate. We will show that there are $\Theta\left(N^{2}\right)$ distinct maximal quasi-progressions contained in $\{1,2, \ldots, N\}$. This will yield an upper bound of $O(\sqrt{N \log N})$ on the discrepancy of quasi-progressions via a standard random coloring argument (see [3]).

Let $F_{N} \doteq\{a / b: 0 \leq a \leq b \leq N ;(a, b)=1\}$ denote the Farey sequence of order $N$. It is well-known (see [7]) that $\left|F_{N}\right| \sim \frac{3 N^{2}}{\pi^{2}}$.

Let $\bar{F}_{N} \doteq\left\{1 / x: x \in F_{N}, x \neq 0\right\}$. Observe that distinct elements of $\bar{F}_{N}$ give rise to quasi-progressions whose restrictions to $\{1,2, \ldots, N\}$ are distinct. Let $\bar{F}_{N}[i]$ denote the $i^{t h}$ smallest element of $\bar{F}_{N}$. Given $\alpha \in(1, N]$, we have $\bar{F}_{N}[t] \leq \alpha<\bar{F}_{N}[t+1]$ for some positive integer $t$.

Suppose there exist integers $s, k \in\{1,2, \ldots, N\}$ such that $s \bar{F}_{N}[t]<k \leq s \alpha$. Then $k / s$, suitably reduced, belongs to $G_{N}$ and lies between $\bar{F}_{N}[t]$ and $\bar{F}_{N}[t+1]$, yielding a contradiction. It follows that $Q(\alpha ; 0, N) \cap\{1,2, \ldots, N\}=Q\left(\bar{F}_{N}[t] ; 0, N\right) \cap\{1,2, \ldots, N\}$. In other words, the number of quasi-progressions contained in $\{1,2, \ldots, N\}$ forms a polynomialsized family, and the $O(\sqrt{N \log N})$ upper bound follows.

It turns out that we can do better, as shown below:

Theorem 5 The hypergraph of quasi-progressions contained in $\{0,1, \ldots, N\}$ has discrepancy $O\left(N^{1 / 3+\varepsilon}\right)$.

Proof Let $q$ be the largest integer such that $N \geq 8 q^{3}$. Let $N=2 A q+B, 0 \leq B<2 q$. Recall that it suffices to consider the elements of $\overline{F_{N}}$, the inverse Farey sequence of order $N$. We divide the set $\{1,2, \ldots, N\}$ into $A$ blocks of size $2 q$, and a residual block of size $B=O\left(N^{1 / 3}\right)$. Each block is colored with precisely one sign change at the halfway mark. Thus either the left half of the block is red and the right half is blue, or vice versa. Observe that the number of terms in the left and right halves of a given block differs by at most one, for any quasi-progression. Thus, with each $\overline{F_{N}}[i]$, we can associate a bias set $B_{i} \subseteq\{1,2, \ldots, 2 A\}$, constructed as follows.

Consider the intersection of the quasiprogression corresponding to $\overline{F_{N}}[i]$ with the $k^{\text {th }}$ block. We mandate that $2 k-1 \in B_{i}$ if and only if the left half of the $k^{t h}$ block contains more elements (in fact, one more element) than the right half, and $2 k \in B_{i}$ if and only if the right half of the $k^{\text {th }}$ block contains more elements than the left half. Clearly, the discrepancy of the hypergraph of quasiprogressions differs from the discrepancy of the hypergraph of these bias sets by at most $O\left(N^{1 / 3}\right)$. Since the latter is a polynomial family of hyperedges over a vertex set of size $O\left(N^{2 / 3}\right)$, random coloring now yields an upper 
bound of $O\left(N^{1 / 3} \sqrt{\log N}\right)$ for the discrepancy of bias sets, and consequently for the discrepancy of quasiprogressions. By increasing the block size by a logarithmic power, the upper bound can, in fact, be improved to $O\left((N \log N)^{1 / 3}\right)$.

Not surprisingly, this argument also beats the random colouring upper bound on the discrepancy of arithmetic progressions. Indeed, Sárközy's $O\left(N^{1 / 3+\epsilon}\right)$ upper bound mentioned in the introduction was founded on quite similar ideas, and had the added advantage of being deterministic, based as it was on properties of quadratic residues.

\section{Acknowledgement}

I thank Professor József Beck for pointers to the literature, useful discussions and constant encouragement. I also thank the referee for several valuable suggestions regarding notation and presentation.

\section{References}

[1] K. F. Roth, On irregularities of distribution. Mathematika 1 (1954), 73-79.

[2] K. F. Roth, Remark concerning integer sequences. Acta Arithmetica 9 (1964), 257260.

[3] P. Erdős and J. Spencer, Probabilistic Methods in Combinatorics. Akadémiai Kiadó, Budapest, 1974.

[4] P. Erdös, On the combinatorial problems which I would most like to see solved. Combinatorica 1 (1981), 25-42.

[5] J. Beck, Roth's estimate of the discrepancy of integer sequences is nearly sharp. Combinatorica 1 (1981), 319-325.

[6] J. Beck, On irregularities of \pm 1 -sequences. Österreich. Akad. Wiss. Math.-Natur. Kl. Sitzungsber II 195 (1986), 13-23.

[7] G. H. Hardy and E. M. Wright, An Introduction to the Theory of Numbers. Clarendon Press, Oxford, 1938.

[8] R. Hochberg, Discrepancy and Bandwidth. Ph.D. Thesis, Rutgers University, 1994.

[9] R. Hochberg, Large discrepancy in homogeneous quasi-arithmetic progressions. Combinatorica 26 (2006), 47-64.

[10] J. Matoušek and J. Spencer, Discrepancy in arithmetic progressions. Journal of the American Mathematical Society 9 (1996), 195-204.

[11] D. Reimer, Five Coloring Theorems. Ph.D. Thesis, Rutgers University, 1997. 